\title{
Predictive Iterative Learning Control with Experimental Validation
}

\author{
Liuping Wang ${ }^{\mathrm{a}}$, Chris T Freeman ${ }^{\mathrm{b}}$, Eric Rogers ${ }^{\mathrm{b}, *}$ \\ ${ }^{a}$ School of Electrical and Computer Engineering, RMIT University, Melbourne, Victoria 3000, Australia \\ ${ }^{b}$ Department of Electronics and Computer Science, University of Southampton, Southampton SO17 1BJ, UK.
}

\begin{abstract}
This paper develops an iterative learning control law that exploits recent results in the area of predictive repetitive control where a priori information about the characteristics of the reference signal is embedded in the control law using the internal model principle. The control law is based on receding horizon control and Laguerre functions can be used to parameterize the future control trajectory if required. Error convergence of the resulting controlled system is analyzed. To evaluate the performance of the design, including comparative aspects, simulation results from a chemical process control problem and supporting experimental results from application to a robot with two inputs and two outputs are given.
\end{abstract}

Keywords: Iterative learning control, predictive control, experimental benchmarking

\section{Introduction}

Many systems complete the same finite duration task over and over again. The sequence is that the task is completed, the system resets to the starting location, the next one is completed and so on. In this paper each execution is termed a trial and the duration is termed the trial length. Once each trial is complete, the system resets to the original location and the next trial can begin, either immediately after the resetting is complete or after a stoppage time has elapsed.

Such systems arise in many industrial applications, where a generic example is a gantry robot undertaking a pick and place task and the sequence of operations is:: i) collect the object from a fixed location, ii) transfer it over a finite duration, iii) place it at a static location or on a moving conveyor, iv) return to the starting location and v) repeat the previous four steps for as many times as required or until a halt is needed for maintenance or other reasons. Similar operations exist in the field of chemical process control such as the operation of batch chemical reactors, see, for example, ((Lee et al., 1996), (Lee et al., 2000), (Lee et al., 2001), (Chin et al., 2004), (Liu et al., 2010)), where the output of the reactor is required to follow a given trajectory over a finite time interval.

Once a trial is complete all information generated during its production is available for use in computing the control signal to be applied on the next trial. Iterative Learning Control (ILC), where the first work is widely credited to (Arimoto et al., 1984), uses information generated on the previous trial, or a finite number thereof, in the computation of the input to be applied on the next trial. The survey papers (Bristow et al., 2006),(Ahn et al., 2007) are a starting point for the literature.

One extensively studied class of ILC laws is based on the minimization of an objective function constructed from the addition of two sums of squares terms and the result summed over the trials, such as Amann et al. (1996), (Lee et al., 2000). The first of these is formed from the current trial

\footnotetext{
${ }^{*}$ Corresponding author

Email addresses: liuping.wang@rmit.edu.au (Liuping Wang), cf@ecs.soton.ac.uk (Chris T Freeman), etar@ecs.soton.ac.uk (Eric Rogers)
} 
error, that is, the difference between the supplied reference signal and the current trial output, and the second from the difference between the control signals used on successive trials, or the current trial signal alone. This class of algorithms is termed, norm optimal, and experimental verification of its performance has also been reported (Ratcliffe et al., 2006; Rogers et al., 2010; Barton and Alleyene , 2011).

This paper develops a predictive ILC design that uses a similar cost function to the one in norm optimal ILC, but embeds the reference signal/disturbance model in the controller and employs the receding horizon control principle. The idea of embedding the reference signal information in the controller has been successfully used in model predictive control, for example, (Wang , 2009) and in other ILC related research (Moore and El-Sharif , 2009). The design allows for the practically motivated case where the reference signal has dominant frequencies and it is decided to only include these in control design as opposed to all frequencies. Also it is assumed that the system dynamics can be adequately modeled, at least for initial control related studies, as linear and time-invariant.

The duration of each trial in ILC is finite and the trial-to-trial error sequence can converge as the number of trials increases even if the system has unstable along the trial dynamics, since over a finite duration only bounded dynamics can be produced. The control design in this paper stabilizes the dynamics on each trial and allows for the rate of convergence to be controlled.

Simulation results from a chemical process control example and supporting experimental data from application of the new results to a two-input two-output robot complete the paper. The next section gives the required background.

\section{Background}

The design in this paper is based on a frequency domain decomposition of the supplied reference signal or vector in the single-input single-output (SISO) and multiple-input multiple-output (MIMO) cases respectively. Once these are selected they are embedded in the process state-space model in accordance with the internal model principle as described next.

Consider the SISO case for simplicity with an obvious generalization to the MIMO case, and suppose that the frequency components of the reference signal to be included in the design have been selected, for details see (Wang et al., 2012),(Wang et al., 2013). This results in the annihilator polynomial

$$
\begin{aligned}
D(z) & =\left(1-z^{-1}\right) \Pi_{i=1}^{l}\left(1-2 \cos (i \omega) z^{-1}+z^{-2}\right) \\
& =1+d_{1} z^{-1}+d_{2} z^{-2}+d_{3} z^{-3}+\ldots+d_{\gamma} z^{-\gamma} .
\end{aligned}
$$

Here 0 and $i \omega, i=1,2, \ldots, l$, for some chosen positive integer $l$, denote the frequencies to be included.

The control law is to be designed to track the reference signal and hence, by the internal model principle (Francis and Wonham, 1975), the corresponding $D(z)$, that is, a particular case of (1) must be included in the denominator of the $z$ transfer-function description of the controller dynamics. In this paper, the method used is to add a vector term $(\mu(p)$ in the state-space model (2) below) to the state dynamics in the plant state-space model as described next, but alternatives exist.

Remark 1. To put this particular design in context, the basic premise is that in many cases the reference signal will have a finite number of dominant frequencies and it suffices to enforce tracking of these frequencies instead of the complete frequency spectrum. This can be viewed as selecting a number of basis functions to approximate the reference signal and there has been other work on such ideas for ILC, see, for example, Sugie and Sakai (2007); van de Wijdeven, and Bosgra (2010); Hamamoto and Sugie (2001). In van de Wijdeven, and Bosgra (2010) the problem considered is that 
the learned command signal is optimal for the specific fixed task only and, in general, extrapolation of the learned command signal to other tasks leads to a significant performance deterioration. Basis functions are used to enhance the extrapolation to a class of reference signals. The approach in Sugie and Sakai (2007); Hamamoto and Sugie (2001) is to restrict the input/output space to an appropriate finite dimensional space spanned by basis functions derived from the reference signal. These are valid alternatives and the question of which one to chose for a given application is discussed again in the last section of this paper.

Suppose that the plant to be controlled has $m_{u}$ inputs and $m_{y}$ outputs and consider the following state-space model at sampling instant $p$,

$$
\begin{aligned}
x_{m}(p+1) & =A_{m} x_{m}(p)+B_{m} u(p)+\mu(p) \\
y(p) & =C_{m} x_{m}(p)
\end{aligned}
$$

where $x_{m}(p)$ is an $n_{1} \times 1$ state vector, $u(p)$ is an $m_{u} \times 1$ input vector and $y(p)$ is an $m_{y} \times 1$ output vector of the plant. Also each entry in the $n_{1} \times 1$ vector $\mu(p)$ is the inverse $z$-transform of $\frac{1}{D(z)}$ and let $q^{-1}$ denote the backward shift operator and $D\left(q^{-1}\right)$ the shift operator interpretation of $D(z)$. Then applying $D\left(q^{-1}\right)$ to $x_{m}(p)$ and $u(p)$ gives

$$
x^{s}(p)=D\left(q^{-1}\right) x_{m}(p), u^{s}(p)=D\left(q^{-1}\right) u(p) .
$$

Also $D\left(q^{-1}\right) \mu(p)=0$ (since $D(z)$ contains all frequencies in $\left.\mu(p)\right)$ and from $(2)$

$$
\begin{aligned}
x^{s}(p+1) & =A_{m} x^{s}(p)+B_{m} u^{s}(p) \\
D\left(q^{-1}\right) y(p+1) & =C_{m} A_{m} x^{s}(p)+C_{m} B_{m} u^{s}(p) .
\end{aligned}
$$

Introducing the state vector

$$
x(p)=\left[\begin{array}{llll}
\left(x^{s}\right)^{T}(p) & y^{T}(p) & \ldots & y^{T}(p-\gamma+1)
\end{array}\right]^{T}
$$

gives the following augmented state-space model for design

$$
\begin{aligned}
x(p+1) & =A x(p)+B u^{s}(p) \\
y(p) & =C x(p) .
\end{aligned}
$$

where

$$
\begin{gathered}
A=\left[\begin{array}{cc}
A_{m} & 0 \\
\hat{C} & A_{d}
\end{array}\right], \hat{C}=\left[\begin{array}{c}
C_{m} A_{m} \\
0
\end{array}\right] \\
A_{d}=\left[\begin{array}{ccccc}
-d_{1} I & -d_{2} I & \ldots & -d_{\gamma-1} I & -d_{\gamma} I \\
I & 0 & 0 & \ldots & 0 \\
0 & I & 0 & \ldots & 0 \\
\vdots & \ddots & \ddots & \ddots & \vdots \\
0 & 0 & \ldots & I & 0
\end{array}\right]
\end{gathered}
$$

and 0 and $I$ denote the zero and identity matrices, respectively, of compatible dimensions $\left(\gamma m_{y} \times\right.$ $\left.\gamma m_{y}\right)$. In addition

$$
\begin{aligned}
B & =\left[\begin{array}{lllllll}
B_{m}^{T} & \left(C_{m} B_{m}\right)^{T} & 0 & \ldots & 0 & 0
\end{array}\right]^{T}, \\
C & =\left[\begin{array}{llllll}
0 & I & 0 & \ldots & 0 & 0
\end{array}\right] .
\end{aligned}
$$

The poles of (4) are the union of those of the system model and those arising from the structure of $\mu(p)$. 


\section{Prediction-based ILC Design}

In the ILC setting $k \geq 0$, is used to denote the trial number and the notation for variables is of the form $y_{k}(p)$ where $y$ is the scalar or vector valued variable under consideration and $p<\infty$ is the number of samples along the trial. The plant dynamics are again described by a state-space model triple. Let $r(p)$ be the supplied reference vector that does not vary from trial-to-trial. Then

$$
e_{k}(p)=y_{k}(p)-r(p)
$$

is the error on trial $k$ and the basic ILC problem is to force the sequence $\left\{e_{k}\right\}$ to converge in $k$.

Suppose that the frequency domain decomposition given in the previous section is applied to $r(p)$ and $D(z)$ of (1), where the latter polynomial is constructed from the frequencies to be included. Then the ILC problem can be formulated by following identical steps to those used to obtain (4), resulting in a state-space model for design of the form

$$
\begin{aligned}
x_{k}(p+1) & =A x_{k}(p)+B u_{k}^{s}(p) \\
y_{k}(p) & =C x_{k}(p)
\end{aligned}
$$

where

$$
x_{k}(p)=\left[\begin{array}{lllll}
\left(x^{s}\right)_{k}^{T}(p) & e_{k}^{T}(p) & e_{k}^{T}(p-1) & \ldots & e_{k}^{T}(p-\gamma)
\end{array}\right]^{T}
$$

and $\left(x^{s}\right)_{k}^{T}(p)$ and $u_{k}^{s}(p)$ are formed using $D(z)$ on trial $k$ as per their counterparts $x^{s}(p)$ and $u^{s}(p)$ in (4). The matrices have identical structures to their counterparts in (4) and by the structure of $C$ the output vector in this state-space model is the current trial error. If it is assumed that the process has reached the steady-state before a trial commences, the state initial vector on each trial can be assumed to be zero.

On trial $k+1$ and sampling instant $p$, the future state vector at sample $p+m$, written $x_{k+1}(p+m)$ $p$ ) for the model (6), is predicted as

$$
x_{k+1}(p+m \mid p)=A^{m} x_{k+1}(p)+\sum_{i=0}^{m-1} A^{m-i-1} B u_{k+1}^{s}(i)
$$

where $m>0$ is a future sampling instant, and this prediction is performed along each trial. In designs that require modeling of the future control trajectory, one approach is to embed an integrator in the design and the incremental control trajectory is then directly computed within an optimization window. For the ILC design considered in this paper, the signal to be optimized is the filtered control vector and the design can be undertaken by modeling this signal using pulse functions. The main drawback is the requirement to optimize a large number of parameters if fast sampling is required and/or the system has a relatively complex dynamic response.

Fast sampling is typically required for mechanical and electro-mechanical systems because the time constants arising in the various sub-components can vary in duration and a smaller sampling interval is required to capture the effects of the smaller of these. One approach to reduce the number of parameters requiring optimization on-line is to parameterize the future trajectory of the filtered control signal using a set of Laguerre functions, where a scaling factor is used to reflect the time scale of the predictive control system.

Laguerre functions have a long standing role in system identification, see, for example, Wahlberg (1991); Heuberger et al. (1995) and their use in model predictive control is detailed in, for example, (Wang , 2004). Within the design developed in this paper, they should only be used in applications where the number of parameters to be optimized is required to be reduced to efficiently construct the control law. This paper considers direct digital control but for cases where the design 
is completed in the continuous-time domain they, or equivalents, must be used to parameterize the trajectories. The following is a summary relevant to the new results in this paper, given for the SISO case with natural MIMO extension noted where required. Also the ILC notation, that is, the trial number subscript, is dropped for ease of presentation.

The basis of the design is the use of a set of discrete orthonormal functions to describe the filtered future control signal $u^{s}(m)$ within a moving horizon window, $0 \leq m \leq N_{p}$. Assume that $N$ is the number of terms in the expansion and let $l_{i}(m), 1 \leq i \leq N$, be a set of Laguerre functions, which are orthonormal. Then

$$
u^{s}(m) \approx \sum_{h=1}^{N} c_{h} l_{h}(m) .
$$

In this application, the $z$ transfer-function of the $h$ th Laguerre function is given by

$$
\Gamma_{h}(z)=\frac{\sqrt{1-a^{2}}}{1-a z^{-1}}\left(\frac{z^{-1}-a}{1-a z^{-1}}\right)^{h-1}
$$

where $0 \leq a<1$ is the scaling factor. Also the network structure of the $z$ transfer-function for this system can be exploited to show that the set of discrete Laguerre functions satisfies the difference equation

$$
L(m+1)=A_{L} L(m)
$$

where $L(m)=\left[\begin{array}{lll}l_{1}(m) & l_{2}(m) & l_{N}(m)\end{array}\right]^{T}$ and

$$
A_{L}=\left[\begin{array}{ccccc}
a & 0 & \ldots & \ldots & 0 \\
\beta & a & \ddots & \vdots & 0 \\
-a \beta & \beta & \ddots & 0 & 0 \\
\vdots & \vdots & \ddots & \ddots & 0 \\
-a^{N-2} \beta & a^{N-3} \beta & \ldots & \beta & a
\end{array}\right]
$$

$\beta=\left(1-a^{2}\right)$ and

$$
L(0)=\sqrt{\beta}\left[\begin{array}{llllll}
1 & -a & a^{2} & -a^{3} & \ldots & (-1)^{N-1} a^{N-1}
\end{array}\right]^{T} .
$$

Setting $a_{h}=0$ and $\delta_{i}(m)=\delta(i)$, where $\delta(i)$ is the Dirac delta function, recovers the standard formulation of model predictive control. The extension to the MIMO case follows immediately on considering each input channel and the corresponding column of the state-space model input matrix.

The basic idea in Laguerre function based design is to represent each input by a set of Laguerre functions together with their unknown coefficients. This is illustrated in the SISO case by

$$
u^{s}(i)=L^{T}(i) \eta
$$

where the Laguerre function vector $L(i)=\left[\begin{array}{llll}l_{1}(i) & l_{2}(i) & \ldots & l_{N}(i)\end{array}\right]^{T}$ and the Laguerre coefficient vector $\eta=\left[\begin{array}{llll}c_{1} & c_{2} & \ldots & c_{N}\end{array}\right]^{T}$. Moreover, $N$ is the dimension of the Laguerre function vector and is also the number of terms used in the approximation. The Laguerre functions are pre-determined in the design once the scaling factor $0 \leq a<1$ and the number of terms $N$ are chosen. In the MIMO case, this procedure is repeated for each input. Moreover, the number of terms and the scaling factor used in this last construction can be chosen independently for each input.

With the control trajectory represented by a Laguerre polynomial, the predicted state vector (8) in the ILC case can be expressed as

$$
x_{k+1}(p+m \mid p)=A^{m} x_{k+1}(p)+\phi^{T}(m) \eta_{k+1}
$$


where $\phi^{T}(m)=\sum_{i=0}^{m-1} A^{m-i-1} B L^{T}(m)$, and this term is invariant from trial-to-trial.

The ILC design is to find the sequence of current trial inputs that minimize the cost function

$$
\begin{aligned}
J & =\sum_{m=1}^{N_{p}} x_{k+1}(p+m \mid p)^{T} Q x_{k+1}(p+m \mid p) \\
& +\sum_{m=0}^{N_{p}}\left(u_{k+1}^{s}(m)-u_{k}^{s}(m)\right)^{T} R\left(u_{k+1}^{s}(m)-u_{k}^{s}(m)\right) .
\end{aligned}
$$

where $Q$ and $R$ are symmetric positive definite matrices and also the difference between the control signals on the current and previous trials is penalized. The motivation for this last choice is to achieve trial-to-trial error reduction without unduly large changes in the amplitudes of the control signals required.

The previous trial filtered input vector is also parameterized in the form detailed above with a long prediction horizon $N_{p}$ and hence

$$
\begin{aligned}
\sum_{m=0}^{N_{p}} u_{k}^{s}(m)^{T} R u_{k}^{s}(m) & =\left(\eta^{k}\right)^{T} R_{L} \eta^{k} \\
\sum_{m=0}^{N_{p}} u_{k+1}^{s}(m)^{T} R u_{k}^{s}(m) & =\left(\eta_{k+1}\right)^{T} R_{L} \eta_{k} \\
\sum_{m=0}^{N_{p}} u_{k+1}^{s}(m)^{T} R u_{k+1}^{s}(m) & =\left(\eta_{k+1}\right)^{T} R_{L} \eta_{k+1}
\end{aligned}
$$

where the orthonormal property of the Laguerre functions has been used, that is, $\sum_{m=0}^{N_{p}} L(m)^{T} L(m)=$ $I$, and $R_{L}$ is an $N \times N$ diagonal matrix.

Substituting (14) and (16)-(18) into (15) gives

$$
\begin{aligned}
J & =\left(\eta_{k+1}\right)^{T} \Omega \eta_{k+1}+2\left(\eta_{k+1}\right)^{T} \Psi x_{k+1}(p) \\
& -2\left(\eta_{k+1}\right)^{T} R_{L} \eta_{k}+\left(\eta_{k}\right)^{T} R_{L} \eta_{k}
\end{aligned}
$$

where

$$
\Omega=\sum_{m=1}^{N_{p}} \phi(m) Q \phi^{T}(m)+R_{L}, \Psi=\sum_{m=1}^{N_{p}} \phi(m) Q A^{m} .
$$

The minimum value of this cost function occurs when

$$
\eta_{k+1}=-\Omega^{-1}\left(\Psi x_{k+1}(p)-R_{L} \eta_{k}\right) .
$$

Under receding horizon control, only the first sample of the optimal control trajectory is implemented, which is constructed in the SISO case with an obvious generalization to MIMO examples, as the filtered control signal on trial $k+1$ at sample $p$

$$
u_{k+1}^{s}(p)=L^{T}(0) \eta_{k+1} .
$$


By combining (20) and (21), the predictive ILC law can be written as

$$
u_{k+1}^{s}(p)=-L^{T}(0) \Omega^{-1} \Psi x_{k+1}(p)+L^{T}(0) \Omega^{-1} R_{L} \eta_{k} .
$$

The control law (22) is the linear sum of terms in the current trial state vector $x_{k+1}(p)$ and the previous trial term $\eta_{k}$. Here the first term introduces state feedback along the trial $k+1$, with constant gain matrix $K_{m p c}$, defined as

$$
K_{m p c}=L^{T}(0) \Omega^{-1} \Psi
$$

and the second term introduces feedforward information from the previous trial. The prediction of the future state at time $\tau$, that is, $x(p+\tau \mid p)$ can be written as

$$
x(p+\tau \mid p)=A^{\tau} x(p)+\phi(\tau) \eta
$$

where

$$
\eta=\left[\begin{array}{llll}
\eta_{1}^{T} & \eta_{2}^{T} & \ldots & \eta_{m_{u}}^{T}
\end{array}\right]^{T}
$$

and if $B_{i}$ denotes the $i$ th column of the state-space model input matrix

$$
\phi(\tau)=\sum_{j=0}^{\tau-1} A^{\tau-j-1}\left[\begin{array}{lll}
B_{1} L_{1}^{T}(j) & \ldots & B_{m_{u}} L_{m_{u}}^{T}(j)
\end{array}\right] .
$$

Also the $i$ th input is given by $L_{i}^{T} \eta_{i}$. where $L_{i}$ is generated by applying (11) for this input. Moreover, the number of terms and the scaling factor used in this last construction can be chosen independently for each input.

In many applications, some entries in the state vector $x_{k}(p)$ given by $(20)$ will not be measurable and in such cases an observer is required. The state vector $x_{k}(p)$ of $(7)$ is formed from $x_{k}^{s}(p)$ and the feedback errors $e_{k}(p), e_{k}(p-1), \ldots, e_{k}(p-\gamma)$, where the latter are measurable and hence it is effective to design a reduced order observer to estimate the plant state dynamics only. Let $\hat{x}_{k}^{s}(p)$ denote the estimated state vector at trial $k$, then the observer dynamics are described by

$$
\hat{x}_{k}^{s}(p+1)=A_{m} \hat{x}_{k}^{s}(p)+B_{m} u_{k}^{s}(p)+K_{o b}\left(y_{k}^{s}(p)-C_{m} \hat{x}_{k}^{s}(p)\right)
$$

where $y_{k}^{s}(p)$ is the filtered output on trial $k$ and sampling instant $p$, defined by $y_{k}^{s}(p)=D\left(q^{-1}\right) y_{k}(p)$. The observer gain matrix $K_{o b}$ is selected such that the closed-loop observer system state matrix $A_{m}-K_{o b} C_{m}$ has all eigenvalues strictly inside the unit circle of the complex plane. After obtaining $\hat{x}_{k}^{s}(p)$, the state vector for the ILC law, $x_{k}(p)$, is constructed using the estimated filtered state vector and the feedback errors as

$$
\hat{x}_{k}(p)=\left[\begin{array}{lllll}
\left(\hat{x}^{S}\right)_{k}^{T}(p) & e_{k}^{T}(p) & e_{k}^{T}(p-1) & \ldots & e_{k}^{T}(p-\gamma)
\end{array}\right]^{T} .
$$

Finally, a fundamental difference between the proposed control action and that of norm optimal ILC (Amann et al., 1996) is that the latter does not have the reference vector embedding (the polynomial $D(z)$ ) in the model for design and hence there is no equivalence even as the Laguerre parameter $N \rightarrow \infty$.

\section{Controlled System Analysis}

In ILC, the trial length is finite and hence trial-to-trial error convergence (that is, in $k$ ) can occur even if the system dynamics are unstable, that is, all eigenvalues of the state matrix do not have 
modulus strictly less than unity. This property is therefore present for ILC dynamics described by (6) and (7) and also for the controlled system under current trial state feedback, as is the case with the design in this paper. The reason for this is that over a finite interval even an unstable linear system can only produce a bounded output.

Unstable dynamics will result in unacceptable along the trial (that is, in $p$ ) dynamics and for lifted model based ILC design, the solution is to first design a stabilizing feedback control law and then apply ILC to the resulting controlled dynamics. Examples of such design can again be found in, for example, the relevant references in the survey papers (Ahn et al., 2007), (Barton and Alleyene, 2011). The analysis below shows that the design of this paper stabilizes the along the trial dynamics and also gives performance information.

As the Laguerre parameter $N$ and the prediction on $p$ increases, it can be shown, see, for example, (Wang , 2009), that control signal (22) can be computed from the solution to the infinite-time discrete quadratic regular problem for the state-space model triple $\{A, B, C\}$ of (7) with symmetric positive definite state and input weighting matrices $Q$ and $R$, respectively. Hence

$$
K_{m p c}=\left(R+B^{T} P_{\infty} B\right)^{-1} B^{T} P_{\infty} A
$$

where $P_{\infty}$ is the solution of the algebraic Riccati equation

$$
A^{T} P_{\infty} A-A^{T} P_{\infty} B\left(R+B^{T} P_{\infty} B\right)^{-1} B^{T} P_{\infty} A+Q-P_{\infty}=0 .
$$

Moreover, by discrete quadratic regulator theory, all eigenvalues of $A-B K_{m p c}$ lie inside the open unit circle in the complex plane.

This last fact, in turn, guarantees that for any matrix norm $\|\cdot\|$ there exist real numbers $M>0$ and $0<\lambda<1$ such that $\left\|\left(A-B K_{m p c}\right)^{p}\right\| \leq M \lambda^{p}, p>0$. Also, for the first trial, with the assumption that $\eta_{-1}$ is a zero vector, the filtered control signal is

$$
u_{1}^{s}(p)=-K_{m p c} x_{1}(p)
$$

and with the control law applied

$$
x_{1}(p+1)=\left(A-B K_{m p c}\right) x_{1}(p)=A_{c l} x_{1}(p)
$$

where $A_{c l}=A-B K_{m p c}$. For a given $x_{1}(0),(29)$ gives

$$
x_{1}(p)=\left(A-B K_{m p c}\right)^{p} x_{1}(0)=A_{c l}^{p} x_{1}(0)
$$

and also

$$
\left\|x_{1}(p)\right\| \leq M \lambda^{p}\left\|x_{1}(0)\right\| .
$$

On the second trial, the filtered control signal is

$$
u_{2}^{s}(p)=-K_{m p c} x_{2}(p)-K_{1} x_{1}(p)
$$

where $K_{1}=L(0)^{T} \Omega^{-1} R_{L} \Omega^{-1} \Psi$, and

$$
x_{2}(p+1)=\left(A-B K_{m p c}\right) x_{2}(p)-B K_{1} x_{1}(p)
$$

or, using (30),

$$
x_{2}(p+1)=\left(A-B K_{m p c}\right) x_{2}(p)-B K_{1}\left(A-B K_{m p c}\right)^{p} x_{1}(0)
$$


or, for given $x_{2}(0)$,

$$
\begin{aligned}
x_{2}(p) & =\left(A-B K_{m p c}\right)^{p} x_{2}(0) \\
& -\sum_{i=0}^{p-1}\left(A-B K_{m p c}\right)^{p-i-1} B K_{1}\left(A-B K_{m p c}\right)^{i} x_{1}(0)
\end{aligned}
$$

and

$$
\begin{aligned}
\left\|x_{2}(p)\right\| & \leq M \lambda^{p}\left\|x_{2}(0)\right\|+\sum_{i=0}^{p-1} M \lambda^{p-i-1}\left\|B K_{1}\right\| M \lambda^{i}\left\|x_{1}(0)\right\| \\
& =M \lambda^{p}\left\|x_{2}(0)\right\|+M^{2} p \lambda^{p-1}\left\|B K_{1}\right\|\left\|x_{1}(0)\right\|
\end{aligned}
$$

By an inductive argument, for trial $k$

$$
\begin{aligned}
\left\|x_{k}(p)\right\| & \leq M \lambda^{p}\left\|x_{k}(0)\right\|+M^{2} p \lambda^{p-1}\left\|B K _ { 1 } \left|\left\|\mid x_{k-1}(0)\right\|\right.\right. \\
& +M^{2} p \lambda^{p-1}\left\|B K _ { 2 } \left|\left\||| x_{k-2}(0)\right\|+\ldots+M^{2} p \lambda^{p-1}\left\|B K _ { k - 1 } \left|\left\|\mid x_{1}(0)\right\|\right.\right.\right.\right.
\end{aligned}
$$

where the gain matrices $K_{1}, K_{2}, \ldots, K_{k-1}$ are bounded from the predictive ILC design. It is seen that the dynamics along each trial are influenced by the state initial vector on this and all previous trials.

In (35) the contributions from the previous trial state initial vectors are critical and their influence decreases as $\lambda$ decreases. One design approach to select a value for $\lambda$, and to place the eigenvalues of $A-B K_{m p c}$ inside a circle with this radius. Such a design also regulates the transient dynamics along the trial by regulating the maximum magnitude that can arise in the response (that is, in $p$ ) produced along any trial. The choice of $\lambda$ is application specific, depending on the form of along the trial dynamics that is required or can be tolerated.

One way to complete this design exercise for the case where the eigenvalues are to be inside a circle of radius $\lambda$ is the following procedure (Wang, 2009).

1. For the selected $0<\lambda<1$, solve the following modified version of the Riccati equation (27) for given symmetric positive definite weighting matrices $Q$ and $R$

$$
\frac{A^{T}}{\lambda} P_{\infty} \frac{A}{\lambda}-\frac{A^{T}}{\lambda} P_{\infty} \frac{B}{\lambda}\left(R+\frac{B^{T}}{\lambda} P_{\infty} \frac{B}{\lambda}\right)^{-1} \frac{B^{T}}{\lambda} P_{\infty} \frac{A}{\lambda}+Q-P_{\infty}=0 .
$$

2. Select $\alpha>1$ such that the matrix $\alpha^{-1} A$ has all eigenvalues inside the unit circle in the complex plane and compute

$$
\gamma=\frac{\lambda}{\alpha}, Q_{\alpha}=\gamma^{2} Q+\left(1-\gamma^{2}\right) P_{\infty}, R_{\alpha}=\gamma^{2} R .
$$

3. For the selected $\alpha$ from the last step, replace the state-space model matrices $A$ and $B$ by $\alpha^{-1} A$ and $\alpha^{-1} B$, respectively. Then complete the design with the cost function

$$
\begin{aligned}
J & =\sum_{m=1}^{N_{p}} x_{k+1}(p+m \mid p)^{T} Q_{\alpha} x_{k+1}(p+m \mid p) \\
& +\sum_{m=0}^{N_{p}}\left(u_{k+1}^{s}(m)-u_{k}^{s}(m)\right)^{T} R_{\alpha}\left(u_{k+1}^{s}(m)-u_{k}^{s}(m)\right)
\end{aligned}
$$

using the $Q_{\alpha}$ and $R_{\alpha}$ of the previous step and a sufficiently large $N_{p}$. 


\section{A Simulation Case Study}

Consider the continuous-time system with transfer-function

$$
G_{p}(s)=\frac{0.8}{(5 s+1)(3 s+1)}
$$

but suppose that the predictive iterative learning controller is designed based on the nominal continuoustime model

$$
G_{m}(s)=\frac{1.2}{(6 s+1)(3 s+1)}
$$

where both plant and model are sampled by a zero-order hold with sampling interval $\Delta t=0.25$ (sec). The system is also subject to a disturbance, which is a filtered square wave signal corrupted with zero-mean normally distributed white noise with variance 0.005 as shown in Figure 1(a). The reference signal is a combination of ramp signals and constants as shown in Figure 1(b).
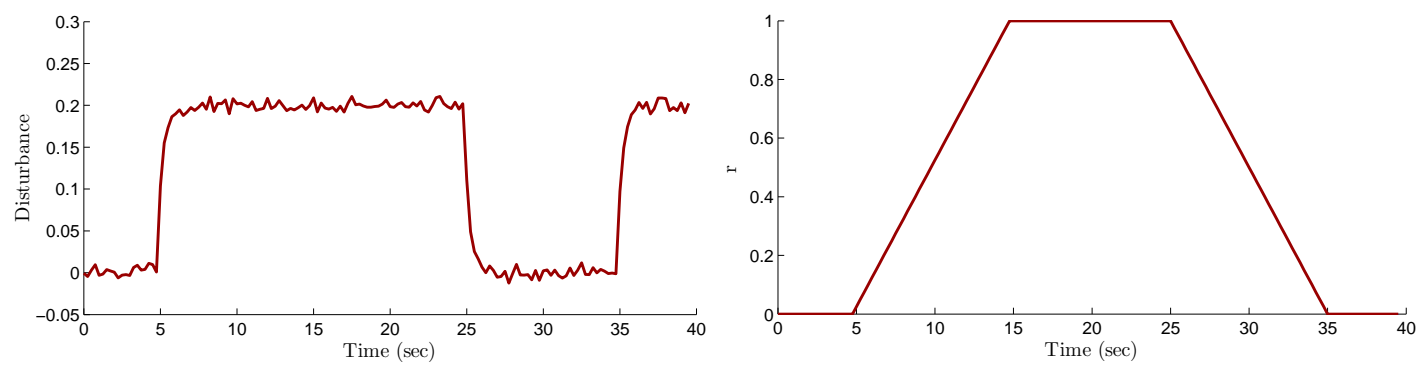

Figure 1: The disturbance (left plot) and reference (right plot) signals used in the simulation.

The $z$-transform of the constant component in the reference signal has the form $\frac{R_{1}}{1-z^{-1}}$ whilst the ramp signal component has the $z$-transform $\frac{R_{2} z^{-1}}{\left(1-z^{-1}\right)^{2}}$ where $R_{1}$ and $R_{2}$ are constants. Moreover, the $z$-transform of the ramp contribution to the reference signal has two poles at $z=1$. Hence if the predictive iterative learning controller is designed to follow a ramp signal, it will automatically follow a constant signal. Therefore, the polynomial $D(z)$ is chosen to be

$$
D\left(z^{-1}\right)=\left(1-z^{-1}\right)^{2}
$$

which is embedded into the model for the design of the prediction-based ILC. The filtered square waved disturbance is assumed to be unknown but its frequency content is known to be concentrated in the low frequency region. Therefore, the selection of $D\left(z^{-1}\right)$ effectively leads to an ILC design that rejects this unknown disturbance.

After sampling, the augmented discrete-time state-space model (state, input and output respectively) matrices of (7) are

$$
A=\left[\begin{array}{cccc}
1.8417 & -0.8465 & 0 & 0 \\
1 & 0 & 0 & 0 \\
0.0082 & -0.0025 & 2 & -1 \\
0 & 0 & 1 & 0
\end{array}\right] ; \quad B=\left[\begin{array}{c}
1 \\
0 \\
0.003 \\
0
\end{array}\right] ; C=\left[\begin{array}{llll}
0 & 0 & 1 & 0
\end{array}\right]
$$

In the exemplar design considered, the Laguerre scaling factor $a=0.35$ and the number of terms $N=10$ were used. Also the weighting matrices in the cost function were $Q=C^{T} C$ and $R=1$ and the eigenvalues of $A-B K_{m p c}$ are required to lie inside the circle of radius $\lambda=0.4$. The design of the corresponding $K_{m p c}$ was undertaken using the Riccati equation based procedure given in the 
previous section. Also the performance measure used in the simulations given next is the sum of the squared errors along each trial, i.e., $E_{k}=\sum_{p}\left(r(p)-y_{k}(p)\right)^{2}$.

Different random seeds were used to generate the noise. A seed of 0 resulted in $E_{1}=0.1526$, $E_{2}=0.1186$ and $E_{n}=0.1191, k \geq 3$. For the random seed chosen as $6, E_{1}=0.147, E_{2}=0.1155$ and $E_{k}=0.1159, k \geq 3$. Many random simulation runs with different seeds to generate the disturbance also resulted in convergence after the third trial. Figure 2 compares the output and reference signals (top plot) and the error (bottom plot) for one case and Figure 3 the corresponding control signal.

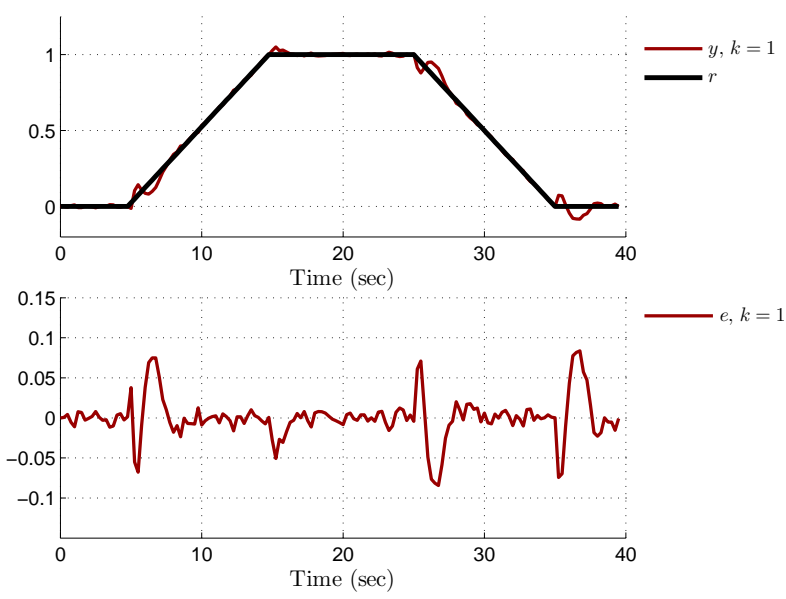

Figure 2: The output $\left(y_{k}\right)$ and error $\left(e_{k}\right)$ produced by the controlled system for trial $k=1$.

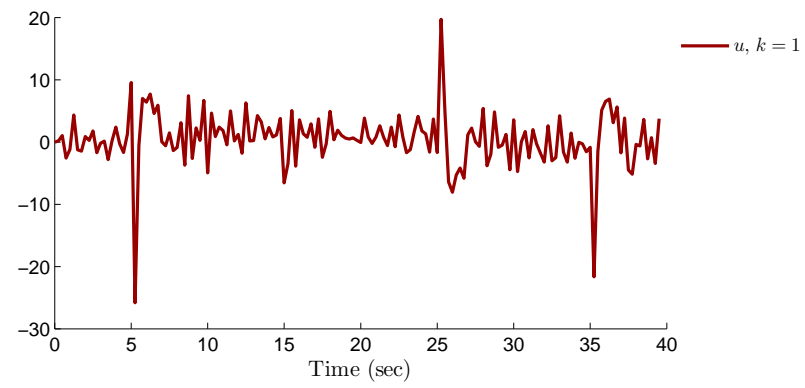

Figure 3: The control signal required by the controlled system.

To examine the effects of the choice of $D(z)$ on performance, a comparative study has been undertaken with the case when $D(z)=1-z^{-1}$, i.e., a single integrator is embedded into the design. In this case $E_{1}=0.953, E_{2}=0.8965$ and $E_{k}=0.8967, k \geq 3$. Figure 4 shows the output and corresponding error after the third trial and Figure 5 the control signal required. In this case there is a small steady-state error in the tracking of the ramp signal, leading to much larger errors.

Figure 6 compares the errors for the alternative designs. It is seen that the algorithm converges after the second trial for both cases. However, for the ILC design with a single integrator embedded, the measure $E_{k}$ is 7.5 times larger (in magnitude) than the case when a double integrator is embedded.

This example was also considered in (Lee et al., 2000) where a $Q$ filter ILC design that implicitly embedded an integrator into the design was given. Hence a detailed comparative aspect should provide much useful information of the relative merits of these two designs given that there is no theoretical answer to which one is better than the other. This also applies to the alternative designs listed under Remark 1 in this paper. 

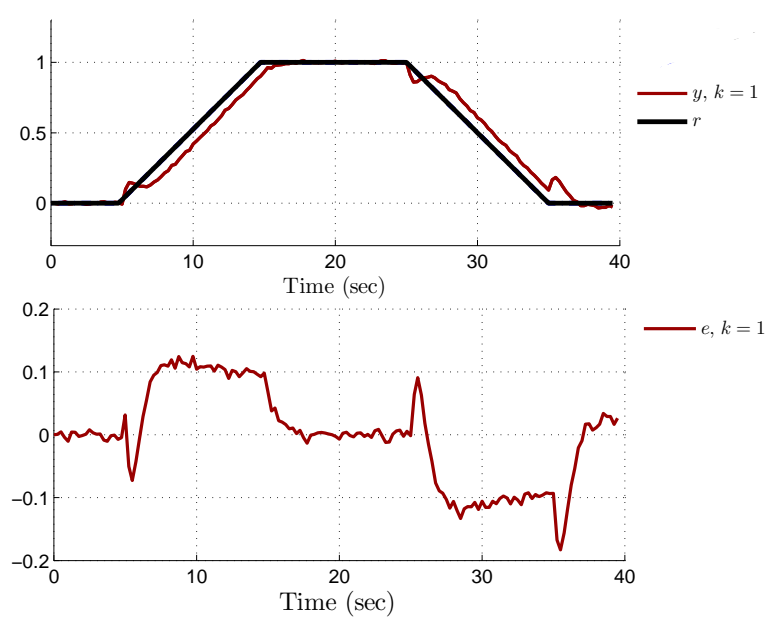

Figure 4: The output $\left(y_{k}\right)$ and error $\left(e_{k}\right)$ produced by the controlled system for trial $k=1$ with $D(z)=1-z^{-1}$.

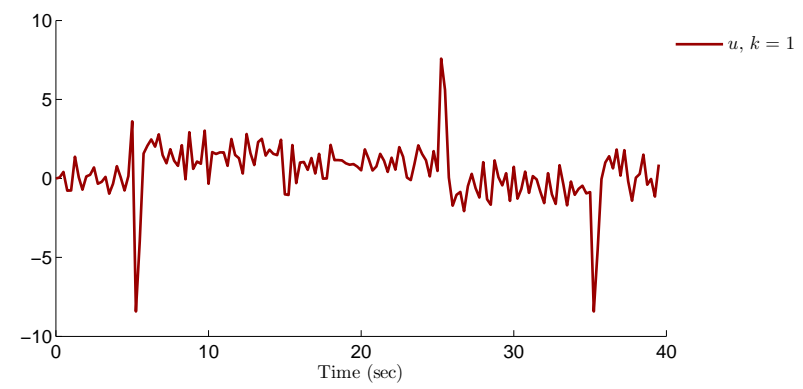

Figure 5: The control signal required by the controlled system with $D(z)=1-z^{-1}$.

\section{An Experimental Case Study}

Figure 7 shows an anthropomorphic robot arm undertaking a 'pick and place' task in a horizontal plane using two joints. The robot end-effector travels from the 'pick' to the 'place' location in a straight line using joint reference trajectories that minimize the end-effector acceleration. During the movement, the arm stops at two intermediate points which are chosen such that there is a change in the direction of travel along the path after reaching each of them. Having reached the 'place' location, the robot repeats the movement in reverse, arriving back at the 'pick' location. Positional and velocity control loops have been implemented around each joint to provide baseline performance and the control scheme operates at $20 \mathrm{~Hz}(\Delta t=0.05 \mathrm{sec})$.

The input and output joint angles have components $u_{i}$ and $y_{i}, i=1,2$, respectively, so that $m_{u}=m_{y}=2$. The dynamics are modelled by the $2 \times 2$ transfer-function matrix

$$
\left[\begin{array}{l}
y_{1}(s) \\
y_{2}(s)
\end{array}\right]=\left[\begin{array}{ll}
G_{11}(s) & G_{12}(s) \\
G_{21}(s) & G_{22}(s)
\end{array}\right]\left[\begin{array}{l}
u_{1}(s) \\
u_{2}(s)
\end{array}\right]
$$

where

$$
\begin{gathered}
G_{11}(s)=\frac{0.16 s^{9}+14.51 s^{8}+578.2 s^{7}+1.392 e 4 s^{6}+2.26 e 5 s^{5}+2.58 e 6 s^{4}+2.09 e 7 s^{3}+1.17 e 8 s^{2}+4.21 e 8 s+7.6 e 8}{5.25 e-5 s^{12}+0.01463 s^{11}+0.91 s^{10}+31.2 s^{9}+714.1 s^{8}+1.19 e 4 s^{7}+1.45 e 5 s^{6}+1.4 e 6 s^{5}+1.01 e 7 s^{4}+5.7 e 7 s^{3}+2.3 e 8 s^{2}+5.9 e 8 s+7.6 e 8}, \\
G_{12}(s)=\frac{-0.022 s^{7}-3.24 s^{6}-88.3 s^{5}-1347 s^{4}-1.06 e 4 s^{3}-4.52 e 4 s^{2}}{5.25 e-5 s^{10}+0.014 s^{9}+0.72 s^{8}+20 s^{7}+363 s^{6}+4645 s^{5}+4.3 e 4 s^{4}+2.9 e 005 s^{3}+1.4 e 6 s^{2}+4.18 e 6 s+6.323 e 6}, \\
G_{21}(s)=\frac{-0.16 s^{7}-8.7 s^{6}-194 s^{5}-2498 s^{4}-1.78 e 4 s^{3}-6.64 e 4 s^{2}}{5.25 e-5 s^{10}+0.014 s^{9}+0.67 s^{8}+17.9 s^{7}+316 s^{6}+3963 s^{5}+3.6 e 4 s^{4}+2.42 e 5 s^{3}+1.1 e 6 s^{2}+3.5 e 6 s+5.3 e 6},
\end{gathered}
$$




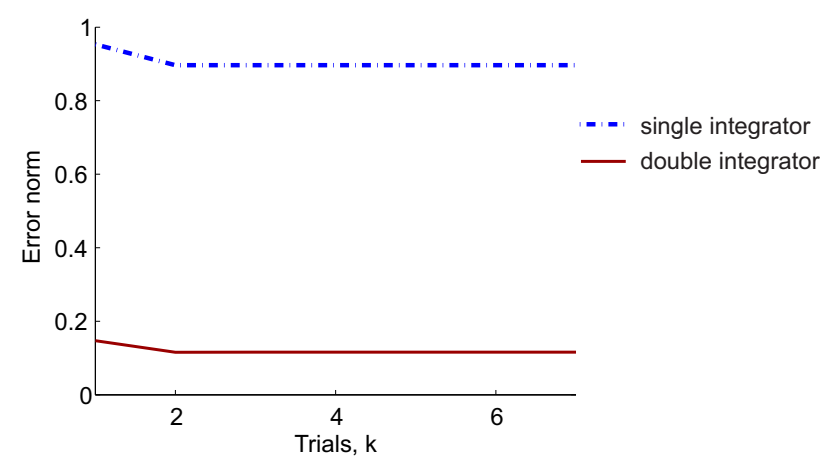

Figure 6: Plot of $E_{k}=\sum_{p}\left(r(p)-y_{k}(p)\right)^{2}$ against the trial number $k$.

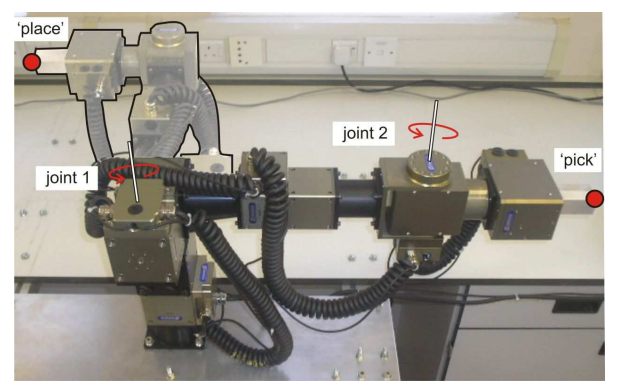

Figure 7: Picture of the robot arm showing the pick and place locations.

$G_{22}(s)=\frac{0.027 s^{9}+4.95 s^{8}+264 s^{7}+7394 s^{6}+1.3 e 5 s^{5}+1.69 e 6 s^{4}+1.5 e 7 s^{3}+9.4 e 7 s^{2}+3.8 e 8 s+7.6 e 8}{5.25 e-5 s^{12}+0.014 s^{11}+0.9 s^{10}+31 s^{9}+714.1 s^{8}+1.19 e 4 s^{7}+1.48 e 5 s^{6}+1.4 e 6 s^{5}+1.04 e 7 s^{4}+5.7 e 7 s^{3}+2.3 e 8 s^{2}+5.9 e 8 s+7.6 e 8}$.

Further details of the robotic system appear in (Wang et al., 2012). Following discretisation, these are expressed by the state-space triple $\left\{A_{m}, B_{m}, C_{m}\right\}$ to produce the required form (2). The task performed by the robot is represented in joint space by the $r_{1}$ and $r_{2}$ reference signals shown in Figure 8. The parameters for the control design are given in Table 1 and the Riccati equation based procedure of Section 4 was used with $\lambda=0.7$ to place all poles of $A-B K_{m p c}$ inside a circle of radius 0.7 . The state observer required for implementation has again been designed as detailed in the previous section with a gain of 100 .

Figure 8 shows the reference signals and the output signals for trials $k=1,2,5$ and confirms that the controlled outputs closely track their reference signals for all three trials. The control signals for the three trials are shown in Figure 9 and the sum of the squared errors for the first five trials also confirms close tracking of the reference signals for each trial as shown in Figure 10. Given the existence of measurement noise in the system and the magnitudes of the errors in Figure 10, it is fair to say that the controlled performance is close to optimal for each trial.

Figure 11 shows the reference signals and output signals for the case where Laguerre term $N_{1}$ $\left(=N_{2}\right)$ is reduced to 6 . It can be seen that this increases output oscillation of both $y_{1}$ and $y_{2}$, leading to the increased error norms shown in Figure 12.

To examine the effect of increasing the weight on the difference between successive control signals in (15), weighting matrix $R$ is modified to $10 \times I$. The Laguerre term $N_{1}\left(=N_{2}\right)$ is returned to its original value of 8 . Figure 13 shows the reference signals and output signals, and indicates that oscillations on outputs $y_{1}$ and $y_{2}$ are reduced compared with those of Figure 8. This improvement in performance is reflected by the reduced error norms shown in Figure 14.

Finally, $R$ is returned to $I$ and the predictive horizon is increased to $N_{p}=400$. Figure 15 shows the reference signals and output signals and shows that again oscillations on both outputs are reduced when compared with Figure 8. The corresponding error norms are shown in Figure 16 and confirm 


\begin{tabular}{|c|r|}
\hline Laguerre pole $a_{1}\left(=a_{2}\right)$ & 0.6 \\
\hline Laguerre term $N_{1}\left(=N_{2}\right)$ & 8 \\
\hline Prediction horizon for each output $N_{p}$ & 100 \\
\hline Weighting matrix $Q$ & $C^{T} C$ \\
\hline Weighting matrix $R$ & $I$ \\
\hline$\alpha$ & 1.1 \\
\hline$\lambda$ & 0.7 \\
\hline
\end{tabular}

Table 1: Design parameters.
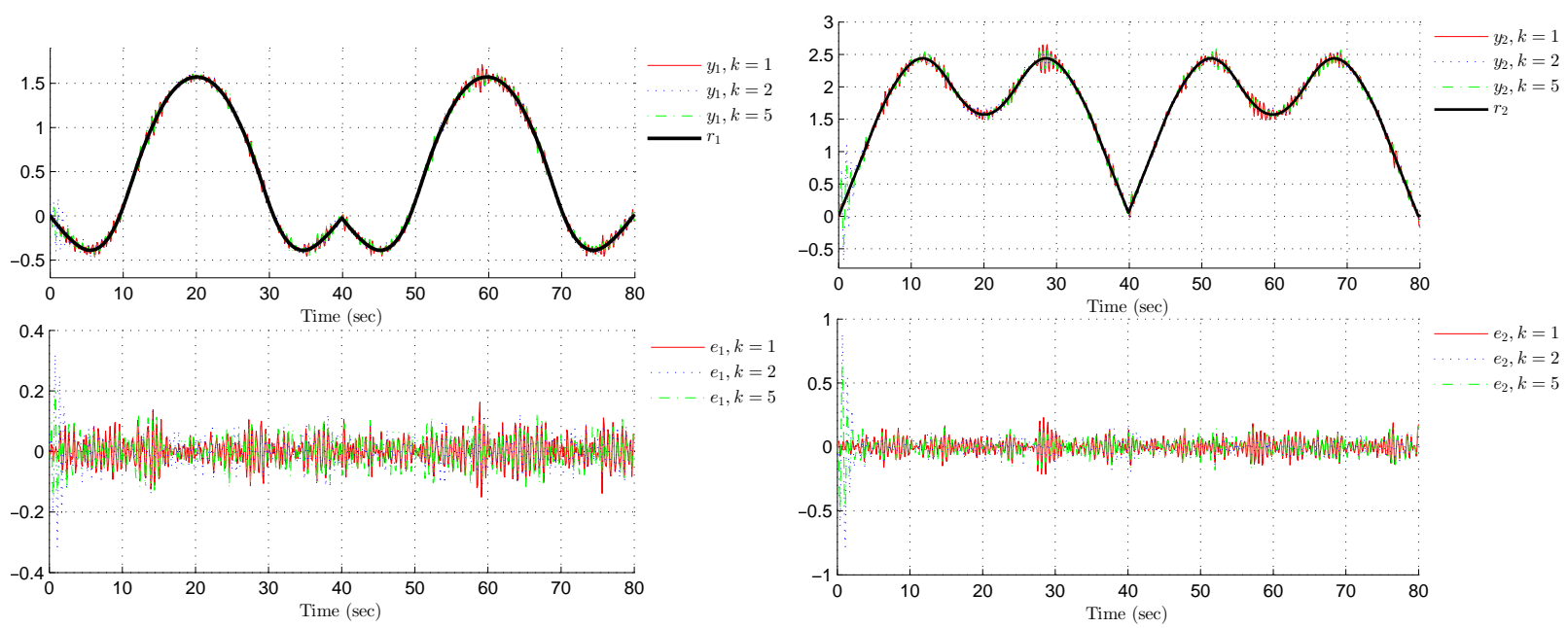

Figure 8: Reference, output and error signals for $k=1,2,5$.

a slight reduction in tracking error. This hence illustrates that a longer prediction horizon embeds higher levels of robustness.

It is also of interest to compare the performance of the new design in this paper with the norm optimal control of, for example, (Amann et al., 1996), where the cost function comprises the addition of two sums of squares terms on each trial and then summed over the trials. The first term is defined by the trial error with symmetric positive definite weighting matrix $Q$, that is, $x_{k+1}^{T}(p) Q x_{k+1}(p)$ and the second by the difference between the control inputs on two successive trials, that is, $\left(e_{k+1}(p)-\right.$ $\left.e_{k}(p)\right)^{T} R\left(e_{k+1}(p)-e_{k}(p)\right)$, where $R$ is a symmetric positive definite matrix.

Figures 17-20 show the experimental results obtained when $Q$ and $R$ correspond to those used in the initial predictive ILC test (i.e., $R=I, Q=C^{T} C$ ). The norm optimal trial-to-trial error convergence is much slower and there are significant disturbances on the output and input.

Finally, the norm optimal weight is adjusted to $Q=100 \times C^{T} C$ in order to provide comparison with a robust inversion based design. Figures 21- 24 show results for this case. It is clear that error norm convergence speed has increased, but is still far lower than the predictive ILC approach proposed in this paper. Tests with alternative $Q$ gains have also been performed and show no increase in convergence speed, confirming that modelling error and disturbance degrade the nominal performance properties of inversion based approaches. Hence these results demonstrate that the new design of this paper has the potential to outperform the norm optimal design.

To provide a comparison against a tuning based design, a phase-lead ILC design has been experimentally tested, i.e., the input for each channel on trial $k+1$ is constructed as

$$
u_{k+1}(p)=u_{k}(p)+\beta e_{k}(p+\lambda)
$$



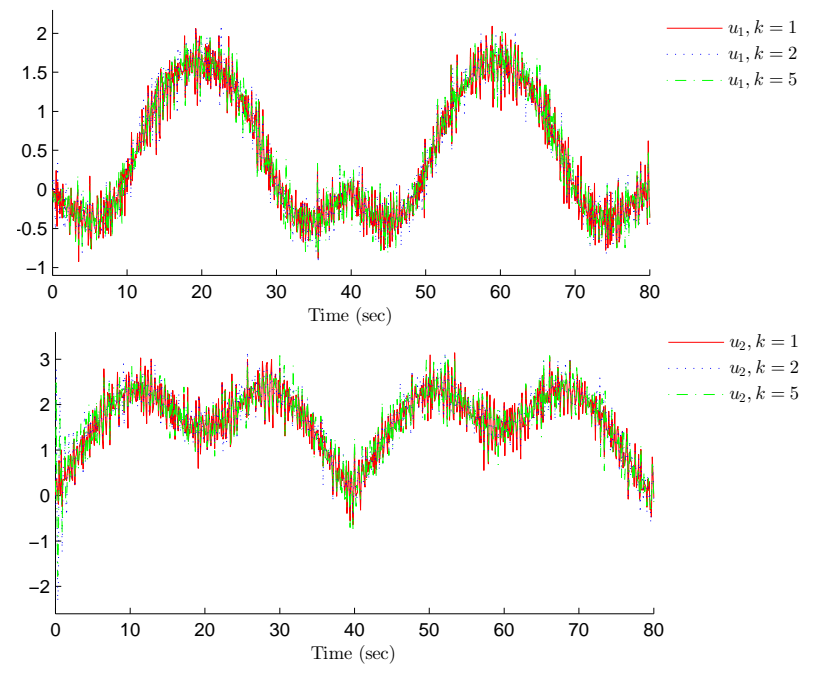

Figure 9: Control signals for $k=1,2,5$.

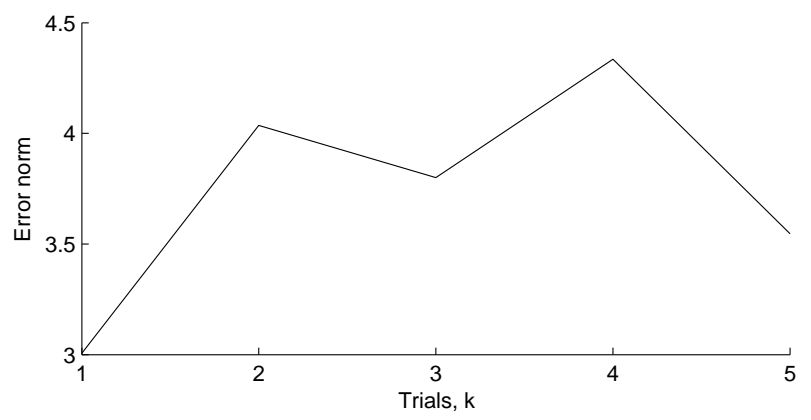

Figure 10: $E_{k}$ for $k=1,2,3,4,5$. 

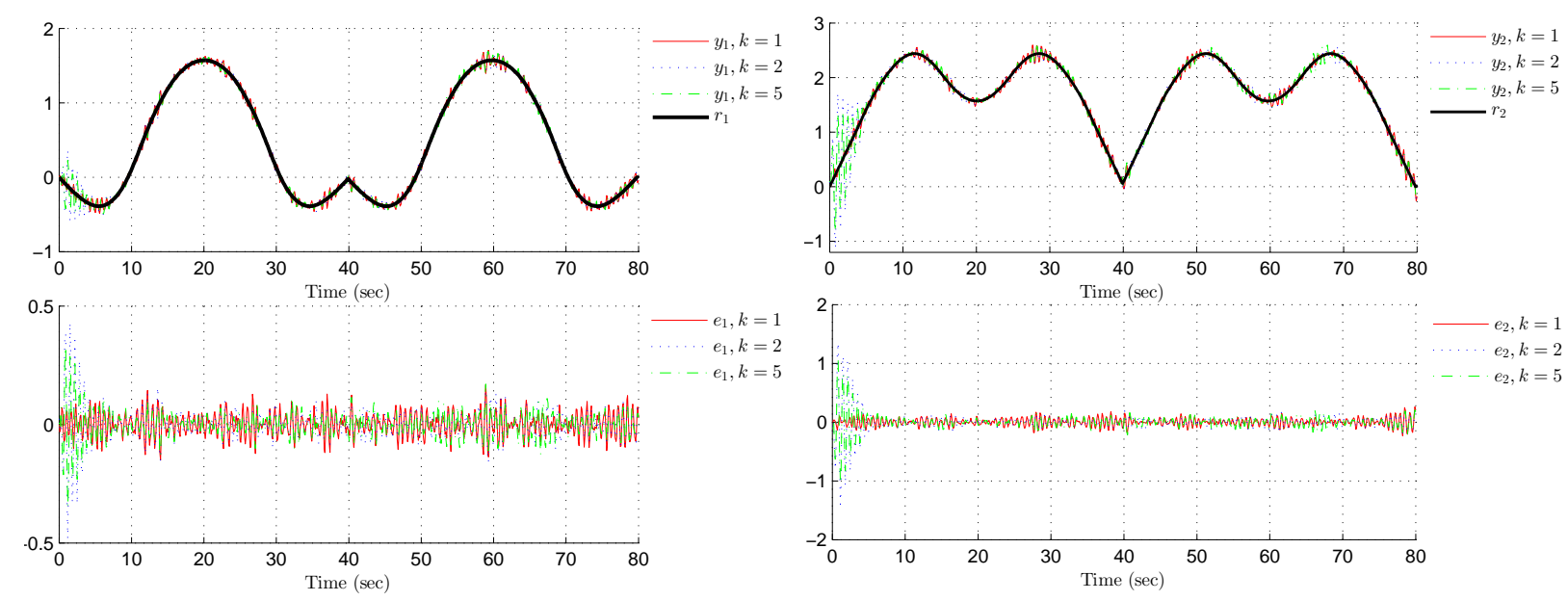

Figure 11: Reference, output and error signals for $k=1,2,5$ using $N_{1}=N_{2}=6$.

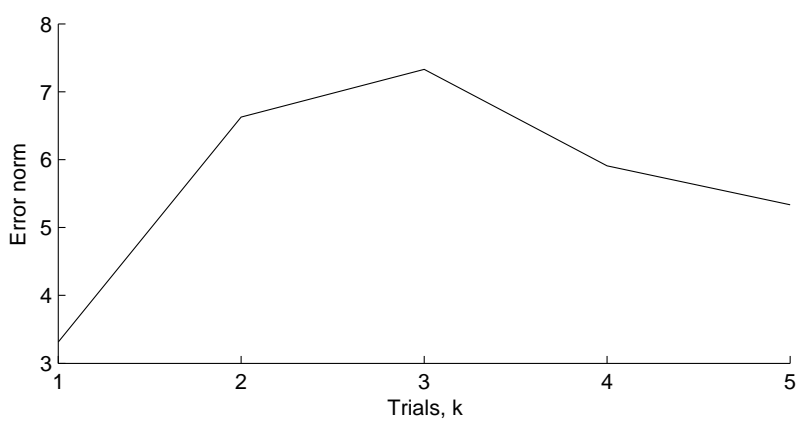

Figure 12: $E_{k}$ for $k=1,2,3,4,5$ using $N_{1}=N_{2}=6$.

where $\beta$ is the gain and $\lambda>0$ is the phase-lead. A series of experiments with different phase- leads was undertaken and the choice of $\lambda=3$ gave the best results, see Figure 25. Overall, these results show that: (1) the lowest possible error norm for any gain or phase-lead value is 1.72 and (2) faster convergence is not possible as instability occurs earlier for the higher gain (i.e., $\beta$ ) required. These results are to be expected given the relatively simple control law structure and the dynamics of this system.

\section{Conclusions}

This paper has developed a new method for predictive iterative learning control design by embedding a prior knowledge about the reference and disturbance signals into the model used for design. It has been verified through simulations and experimental studies that use of this information can result in fast convergence of the error and better performance from the controlled system. The control design is based on the receding horizon principle and a natural area for further research is to impose hard constraints on, for example, the inputs and outputs. Also there is the need for in depth comparative studies to benchmark this design against alternatives with supporting experimental evidence. An obvious goal of such studies would be to compare this design method against, in particular, the others listed under Remark 1. Given that there will be no universal best choice for all applications another aim would be to develop guidelines as to which method gives the best results for a given application. 

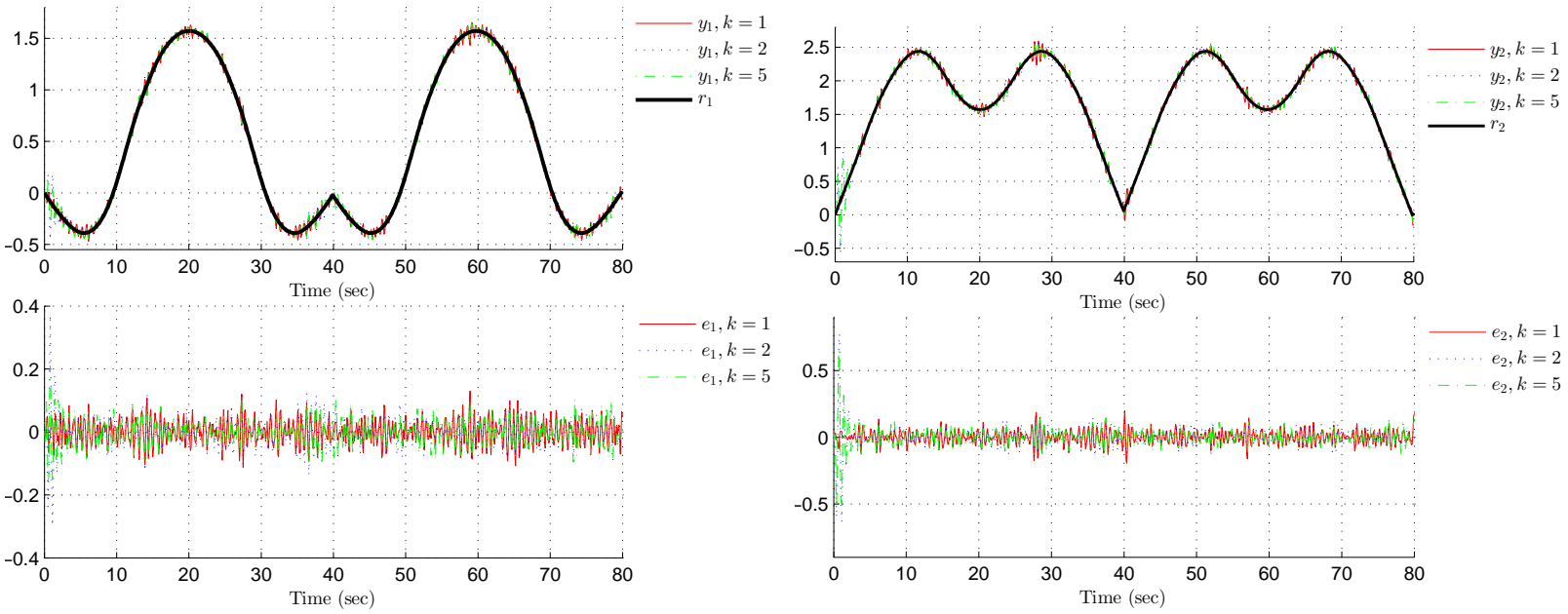

Figure 13: Reference, output and error signals for $k=1,2,5$ using $N_{1}=N_{2}=8$ and $R=10 \times I$.

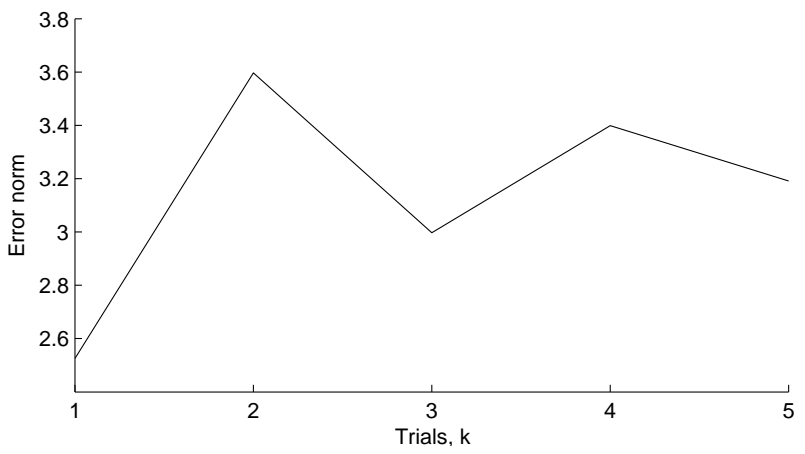

Figure 14: Sum of squared error signals of $k=1,2,3,4,5$ using $N_{1}=N_{2}=8$ and $R=10 \times I$.

\section{References}

Ahn, H., S., Chen, Y., and Moore, K. L. (2007) "Iterative learning control: brief survey and categorization", IEEE Transactions on Systems, Man and Cybernetics, Part C, 2007, 37(6), 1109-1121.

Amann, N., Owens, D. H., and Rogers, E. (1996) "Iterative learning control for discrete-time systems with exponential rate of convergence", Proceedings of The Institution of Electrical Engineers, Pt D, 143(2), 217-224.

Arimoto, S., Kawamura, S., and Miyazaki F. (1984) "Bettering the operation of robots by learning," Journal of Robotic Systems, 1, 123-140.

Barton, K. L., and Alleyne, A. G. (2011) "A norm optimal approach to time-varying ILC with application to a multi-axis robotic testbed," IEEE Transactions on Control Systems Technology, 19(1), 166-180.

Bristow, D. A., Tharayil, M., and Alleyne, A. G. (2006), "A survey of iterative learning control: a learning-based method for high-performance tracking control" IEEE Control Systems Magazine, $26(3), 96-114$.

Chin, I., Qin, S. J., Lee, K. S., and Cho, M. A. (2004) "Two-stage iterative learning control technique 

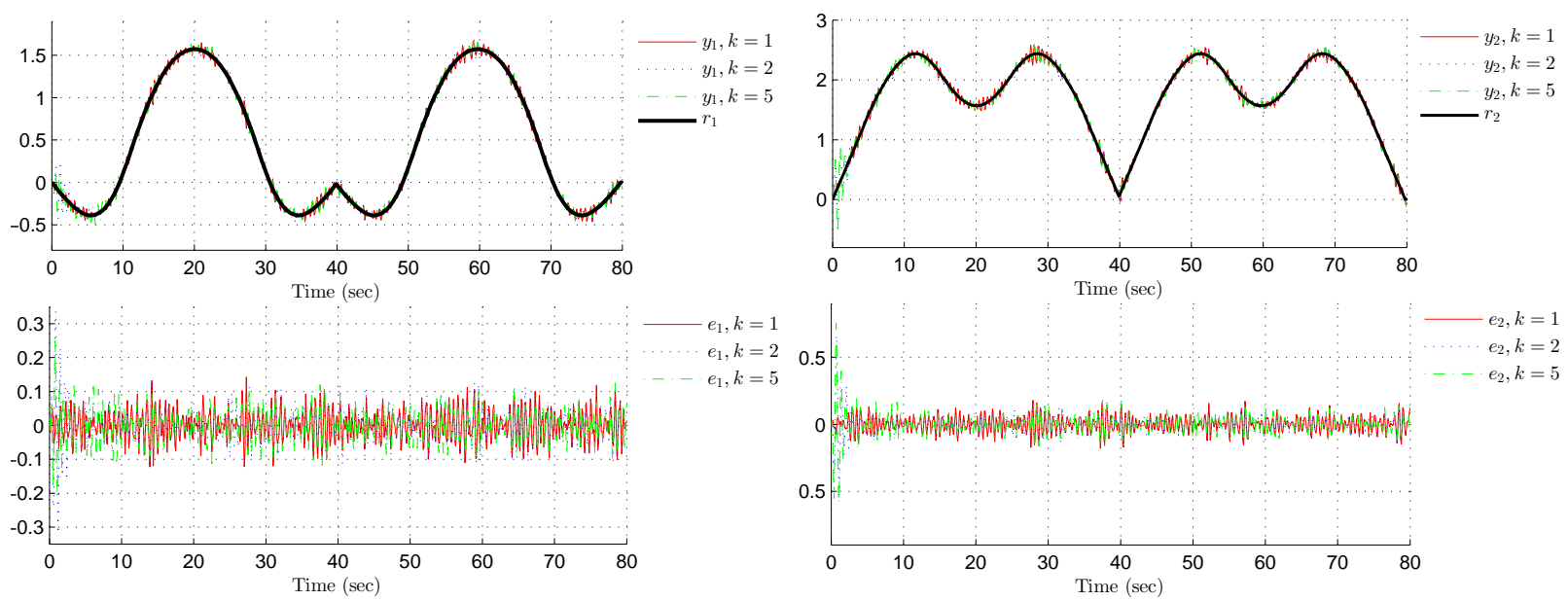

Figure 15: Reference, output and error signals for $k=1,2,5$ using $N_{1}=N_{2}=8$ and $N_{p}=400$.

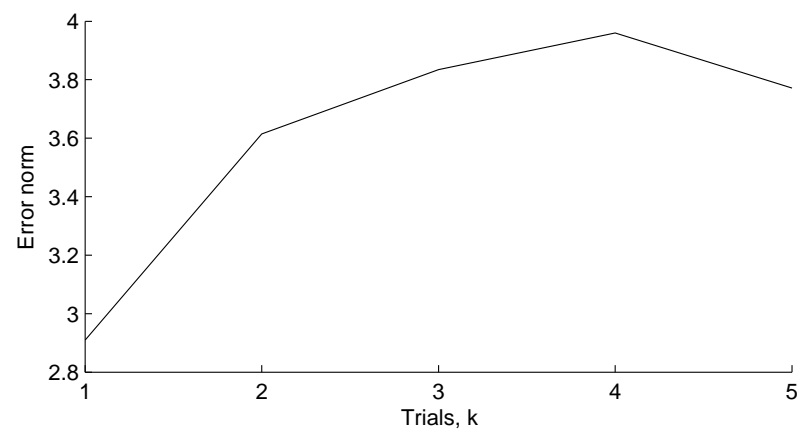

Figure 16: Sum of squared error signals of $k=1,2,3,4,5$ using $N_{1}=N_{2}=8$ and $N_{p}=400$.

combined with real-time feedback for independent disturbance rejection," Automatica, 40, 19131922.

Francis, B. A., and Wonham, W. M. (1975) "The internal model principle for linear multivariable regulators," Journal of Applied Mathematics and Optimization, 2, 170-194.

Hamamoto, K., and Sugie, T. (2001) "An iterative learning control algorithm with prescribed inputoutput space," Automatica, 37(11), 1803-1809.

Heuberger, P. S. C., van den Hof, P. M. J., and Bosgra, O. H. (1995) "A generalized orthonormal basis for linear dynamics systems," IEEE Transactions on Automatic Control, 40(3), 451-465.

Lee, K. S., Bang, S. H., Yi, S., Son, J. S., and Yoon, S. C. (1996) "Iterative learning control of heat-up phase for a batch polymerization reactor," Journal of Process Control, 6, 255-262.

Lee, J. H., Lee, K. S., and Kim, W. C. (2000) "Model-based iterative learning control with a quadratic criterion for time-varying linear systems," Automatica, 36, 641-657.

Lee, J. H., Natarajan, S., and Lee, K. S. (2001) "Model-based predictive control approach to repetitive control of continuous process with periodic operations," Journal of Process Control, 11, 195-207.

Liu, T., Gao, F., and Wang, Y. (2010) "IMC-based iterative learning control for batch process with uncertain time delay," Journal of Process Control, 20, 173-180. 


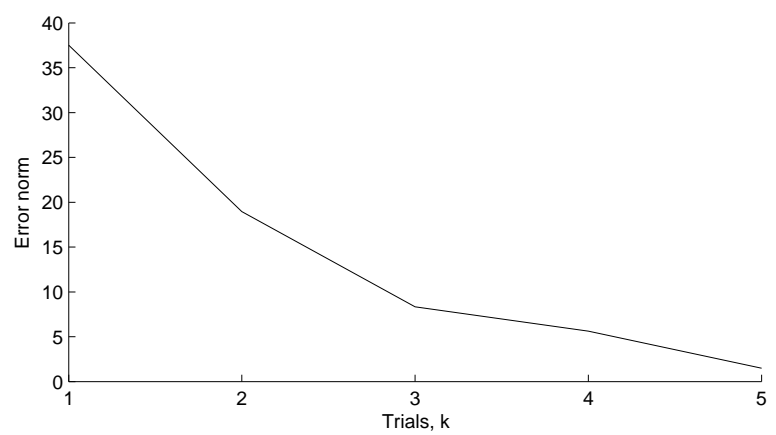

Figure 17: Sum of squared error for the norm optimal design with $R=I$ and $Q=C^{T} C$.

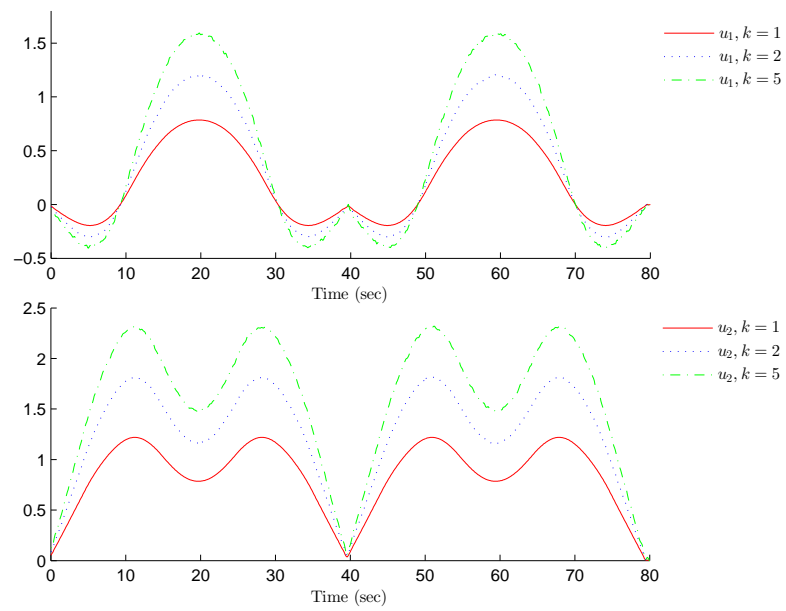

Figure 18: Control input signals for the norm optimal design with $R=I$ and $Q=C^{T} C$.
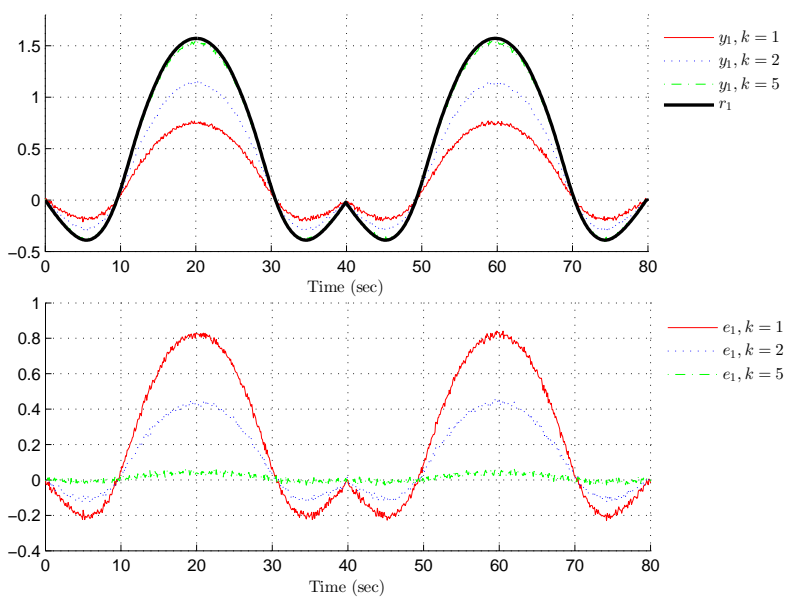

Figure 19: Reference, output and error signals for output $y_{1}$ in the norm optimal design with $R=I$ and $Q=C^{T} C$. 

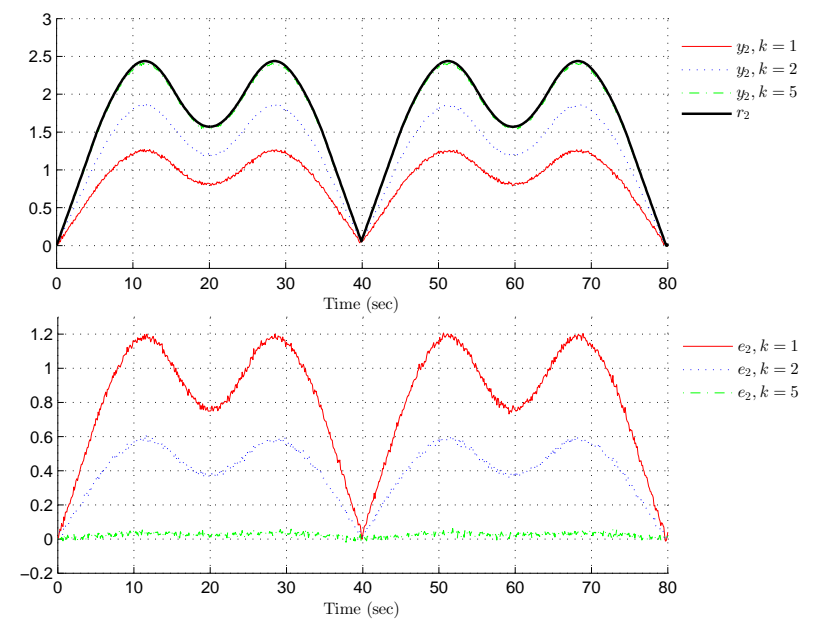

Figure 20: Reference, output and error signals for output $y_{2}$ in the norm optimal design with $R=I$ and $Q=C^{T} C$.

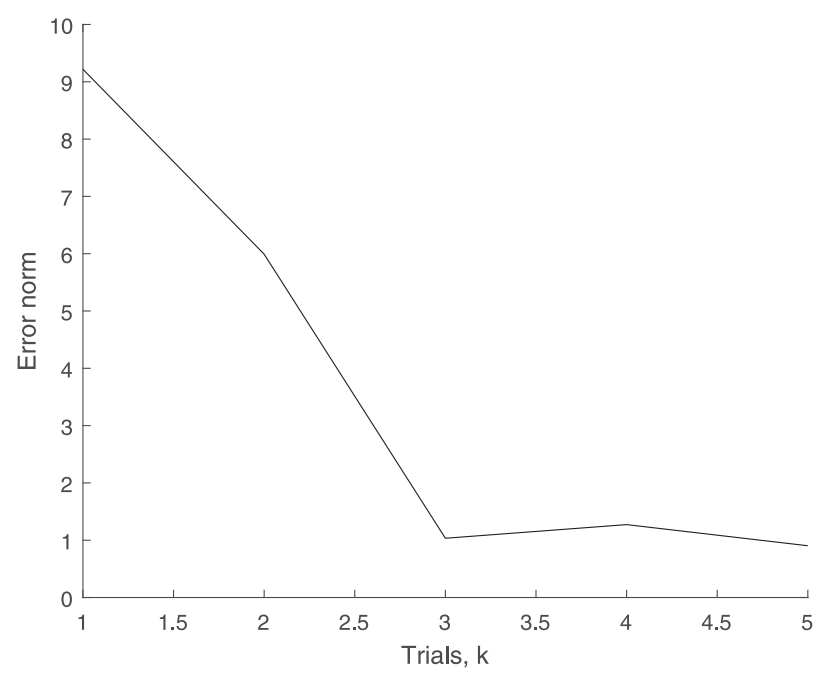

Figure 21: Sum of squared error for the norm optimal design with $R=I$ and $Q=100 \times C^{T} C$.
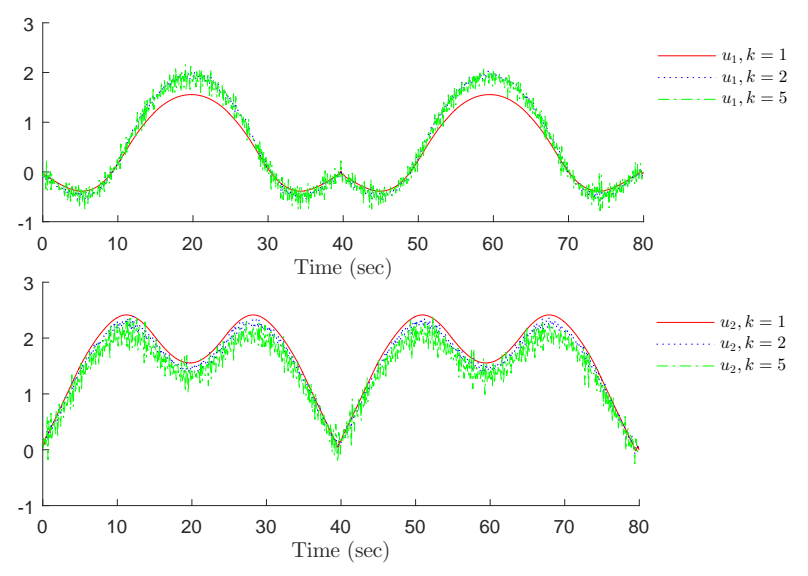

Figure 22: Control input signals for the norm optimal design with $R=I$ and $Q=100 \times C^{T} C$. 

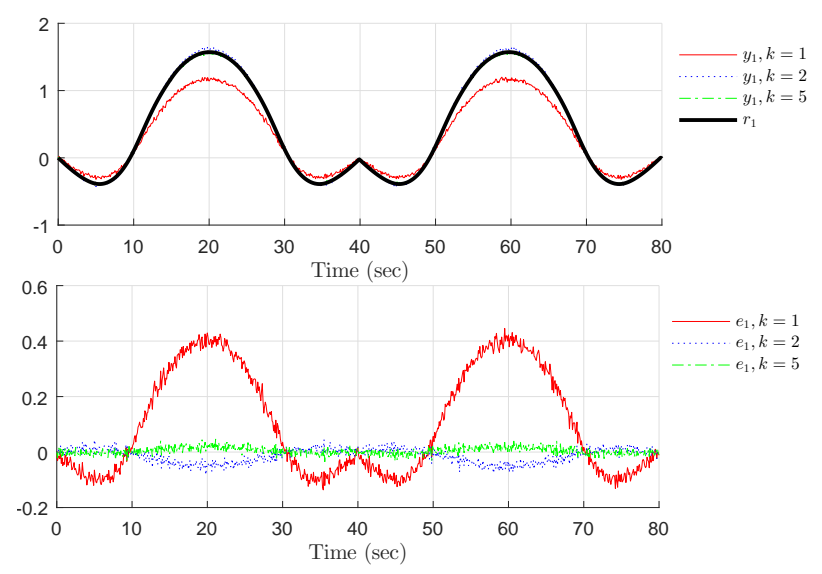

Figure 23: Reference, output and error signals for output $y_{1}$ in the norm optimal design with $R=I$ and $Q=100 \times C^{T} C$.
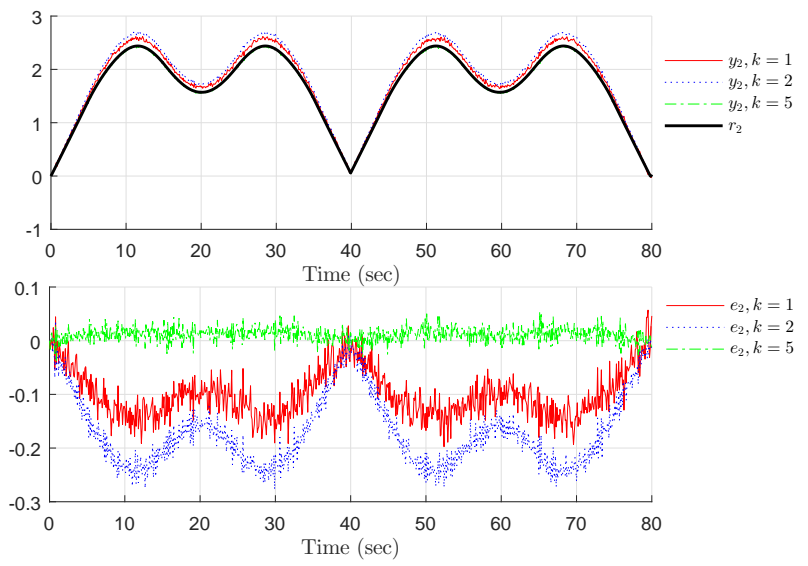

Figure 24: Reference, output and error signals for output $y_{2}$ in the norm optimal design with $R=I$ and $Q=100 \times C^{T} C$.

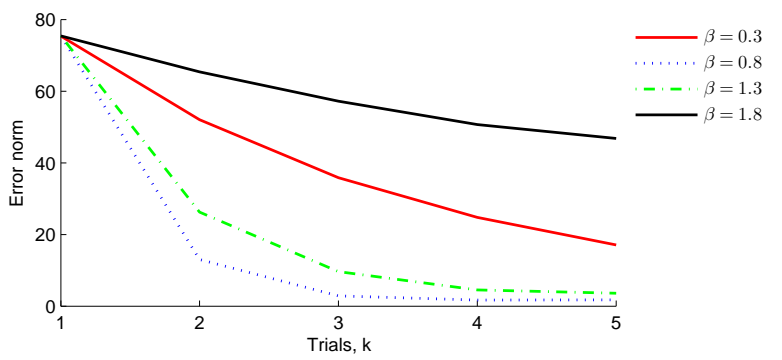

Figure 25: Sum of squared error for the phase-lead ILC design (40) with $\lambda=3$ and various $\beta$ gains. 
Moore, K. L., El-Sharif, O. (2009) "Internal model principle for discrete repetitive processes." Proceedings of the 48th IEEE Conference on Decision and Control, 446-451.

Ratcliffe, J. D., Lewin, P. L, Rogers, E., Hatonen, J. J., and Owens, D. H. (2006) "Norm optimal iterative learning control applied to gantry robots for automation applications," IEEE Transactions on Robotics, 22(6), 1303-1307.

Rogers, E., Owens, D. H., Werner, H., Freeman, C. T., Lewin, P. L., Kichhoff, S., Schmidt, C., and Lichtenberg, G. (2010) "Norm optimal iterative learning control with application to problems in accelerator based free electron lasers and rehabilitation robotics," European Journal of Control, 16(5), 497-524.

Sugie, T., and Sakai, F. (2007) "Projection based iterative learning control with its application to continuous-time idenitificaiton," Processings of ICKS, 95-102.

van de Wijdeven, J. and Bosgra, O. H. (2010) "Using basis functions in iterative learning control: analysis and design theory," International Journal of Control, 83(4), 661-675.

Wahlberg, B. (1991) "System identification using Laguerre models," IEEE Transactions on Automatic Control, AC-36, 551-562.

Wang, L. (2004) "Discrete model predictive control design using Laguerre functions," Journal of Process Control, 14, 131-142.

Wang, L. (2009) "Model Predictive Control Systems Design and Implementation using MATLAB," Springer, London.

Wang, L., Gawthorp, P. J., Owens, D. H., and Rogers, E. (2010) "Switched linear predictive controllers for periodic exogenous signals." International Journal of Control, 83(4), 848-861.

Wang, L., Chai, S., Rogers, E., and Freeman, C. T. (2012) "Multivariable repetitive-predictive controllers using frequency decomposition," IEEE Transactions on Control Systems Technology, 20(6), 1597-1604.

Wang, L., Freeman, C. T., Chai, S., and Rogers, E. (2013) "Predictive-repetitive control with constraints: from design to implelemntation." Journal of Process Control, 23(7), 956-967. 